DOI:

10.1038/nrn2289

NEUROLOGICAL DISORDERS

\title{
Stimulating side effects
}

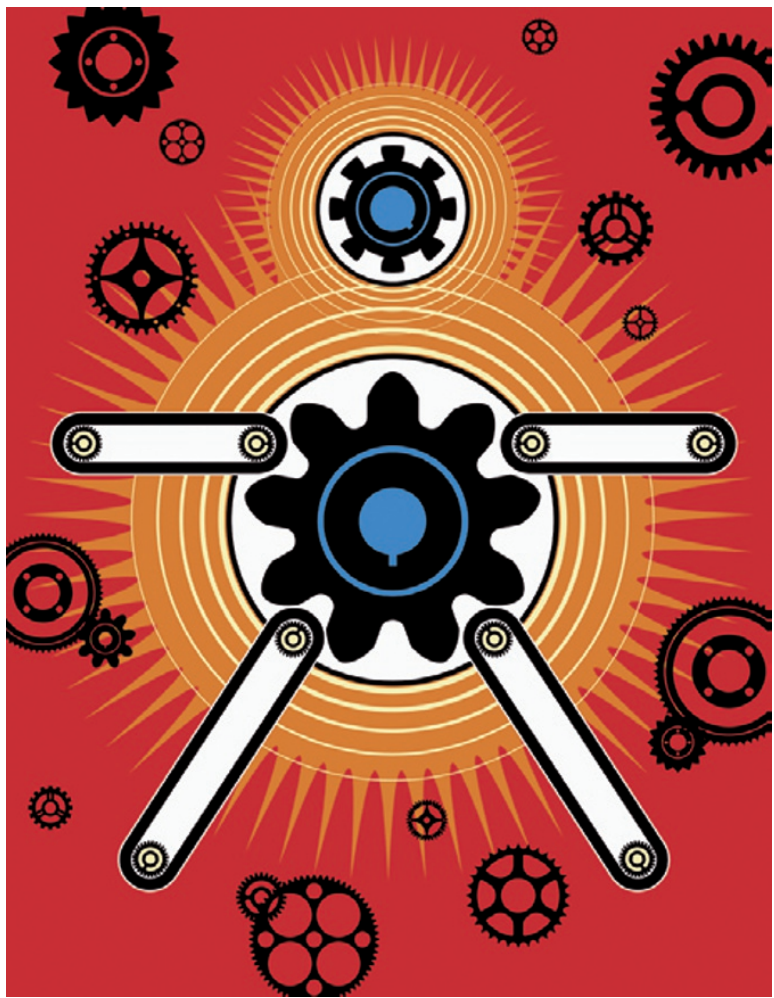

The motor symptoms of Parkinson's disease can be reduced by deep-brain stimulation (DBS) of the subthalamic nucleus (STN). Unfortunately, in some patients this treatment produces psychiatric side effects including depression and impulsivity. Two recent studies have provided insight into the mechanisms that underlie these side effects.

Frank et al. used a decisionmaking task to show that healthy individuals and patients with Parkinson's disease taking dopaminergic medication take longer to make difficult decisions that involve a high degree of conflict than to make easy decisions. By contrast, patients with Parkinson's disease undergoing DBS of the STN responded faster in high-conflict choices, and also made more incorrect decisions. This DBSinduced premature responding was reversed when the stimulator was switched off. Thus, DBS of the STN might increase impulsivity by reducing the time that is taken to make high-conflict decisions.

In a different study, Sharp and colleagues showed that STN stimulation inhibited the firing rate of serotonin neurons in the dorsal raphe nucleus (DRN) in normal rats, as well as in two rat models of Parkinson's disease. They then went on to show that infusion of a $\mathrm{GABA}_{\mathrm{A}}$ $(\gamma$-aminobutyric acid) receptor agonist into the STN produced the same result, indicating that a stimulationinduced reduction in STN activity might cause the inhibition of DRN serotonin neurons. STN stimulation also induced depression-like behaviour in the rats, causing 'behavioural despair' in the forced-swim test. This effect was inhibited by treatment with a selective serotonin reuptake inhibitor, providing additional evidence that the depressive effect of STN stimulation might be due to the inhibition of serotonergic neurotransmission.

Interestingly, reduced serotonin function has also been associated with increased impulsivity, and although it is still unclear whether the results from the second study translate to humans, this suggests that the inhibition of serotonin cell firing that is induced by stimulation of the STN might underpin both the depression and the increased impulsivity that is seen in patients with Parkinson's disease undergoing this treatment.

Leonie Welberg

ORIGINAL RESEARCH PAPERS Frank, M. J. et al.

Hold your horses: impulsivity, deep brain stimulation, and medication in Parkinsonism. Science 25 Oct 2007 (doi:10.1126/ science.1146157) | Temel, Y. et al. Inhibition of 5-HT neuron activity and induction of depressivelike behavior by high-frequency stimulation of the subthalamic nucleus. Proc. Natl Acad. Sci. USA 104, 17087-17092 (2007) 